\title{
ANALYSIS OF SULPHUR CONTENT IN COPRA
}

\author{
A. Stephen Sagayaraj, G. Ramya and N. Dhanaraj \\ Department of Electronics and Communication Engineering, Jai Shriram Engineering College, India
}

\begin{abstract}
Agriculture is the largest economic sector in India. Coconut is one of the most demanded fruit amongst all. The dried coconut, copra is the main source of coconut oil. Naturally it contains $70 \%$ of moisture content and it is dried to about $7 \%$ for production of coconut oil. The sulphur is added as preservative which acts as anti-microbial agent for preventing bacteria, fungus etc. Sulphur is a toxic food preservative which restricts lung performance and leads to direct allergenic reactions. The survey of World Health Organisation says that 65\% of asthmatic children are sensitive to sulphur and $75 \%$ of children exposed to sulphur exhibits changes in their behaviour. The sulphur fumigation over coconut affects human both externally and internally. Fumigation leads to cancer and environmental pollution. In order to prevent this devastating effect, copra is examined using image processing. The proposed idea is to identify the presence and percentage of sulphur region present in copra. The region of interest is segmented by method of superimposition thereby segmenting white layers in copra. The RGB colour features are extracted to differentiate the sulphur added copra from normal copra. The coconut is dried under $60^{\circ} \mathrm{C}$ in a tray drier and shapes of copra decreases at regular interval of time are extracted using image processing. The decreasing percentage of shape features are measured to identify the sulphur added in the copra. The k-means clustering technique is used to discriminate the copra at different levels. The segmented patch area is measured to determine the percentage of sulphur present in copra. The percentage of sulphur over copra is divided into three levels (low sulphur added region, medium sulphur added region and high sulphur added region). The K-Nearest Neighbour classification is also used to classify the sulphur added copra at different levels. The proposed algorithm classifies the sulphur added copra at three different levels with $86 \%$ accuracy.
\end{abstract}

Keywords:

Fumigation, RGB, k-means Clustering, KNN

\section{INTRODUCTION}

Agriculture is one of the largest economic sectors, which plays a major role in economic development of our country. There is an increasing demand of coconut and its products. Coconut is enriched with more vitamins and necessary nutrients essential for human bodies. It is commonly consumed in daily diets and acts as a major source of anti-oxidant. India is the third largest coconut producing country (area-15.5\%, coconut production-21\%) in the world. It annually produces 14.81 billion nuts from an area of 1.93 million ha. Copra is a richest source of coconut oil (70\%). Moisture content (53.84\%, wet basis) in fresh coconuts is required to be reduced to $7 \%$ by drying to concentrate oil content. There would be contamination by insects, birds, animals and dust thereby leading to spoilage and degradation of the quality of coconuts. If the drying atmosphere is highly humid, there is an enough possibility of fungal and bacterial infections over the products. In order to avoid those infections, farmers used the process of sulphur fumigation over the coconuts so as to keep the coconuts in a good condition, ready to be sold out to the merchants.

Sulphur is used as a preservative in dried fruit to prevent it from spoiling and also to preserve the fruit's bright colour. The sulphur dioxide is a gas that's created by burning coal or oil. Breathing sulphur gas is toxic, and too much exposure can cause serious illness or possibly even death. Asthma, Emphysema, Chronic Bronchitis, and respiratory diseases are caused by sulphur. People in taking foods treated with this gas, may experience skin rashes, an upset stomach, or even an asthma attack.

The characteristics of fruits are different in various moisture conditions. The variation in drying condition of fruit is measured [1]. The colour and texture are measured for recognition of fruits. The functionality and flexibility is improved by measuring color and texture features [2]. Deepa et al. [4] provided the comparative analysis of GLCM, intensity and shape based features and measured the performance using PNN. The quality inspection and grading of mangoes is done by measuring shape and size features [7]. The local binary patterns are used to detect and classify the apple [12]. Neural networks are used to analyse the diseases of fruits. The efficiency of detecting diseases of fruits is high by using neural networks [13] [15]. In advance adaptive probabilistic neural network and Adaptive general regression neural network is used to improve the efficiency [14]. The FPGA implementation of image processing using XSG to improve the classification efficiency [10] [6].

The proposed block diagram is illustrated in section 2. The segmentation of ROI is discussed in section 3. The RGB colour extraction and colour histogram of the image is discussed in section 3. The shape feature extraction of dried copra at regular interval of time is discussed in section 4 .

\section{METHODOLOGY}

The proposed method gives deep explanation about sulphur fumigated copra and percentage of sulphur fumigated over copra. The coconut is dried under two conditions:

- Drying under normal condition (Direct sunlight drying without presence of sulphur) and

- Drying under presence of sulphur (Direct sunlight drying with presence of sulphur in fumigated condition).

The Fig.1 explains the methodology for identifying sulphur fumigated copra in coconut industry to avoid the adulteration. The Region of interest is obtained by eliminating the copra's brown colour shell (i.e. only white layers are isolated). Initially, after finding out ROI, the colour and shapes are extracted to detect the presence of sulphur. Once the algorithm detects the presence of sulphur $k$-means clustering is used to segment the Sulphur patch present in the copra. The white pixel is to measure the percentage 
of sulphur present in the copra. KNN Classifier is used to measure the accuracy of sulphur fumigated and normal copra.

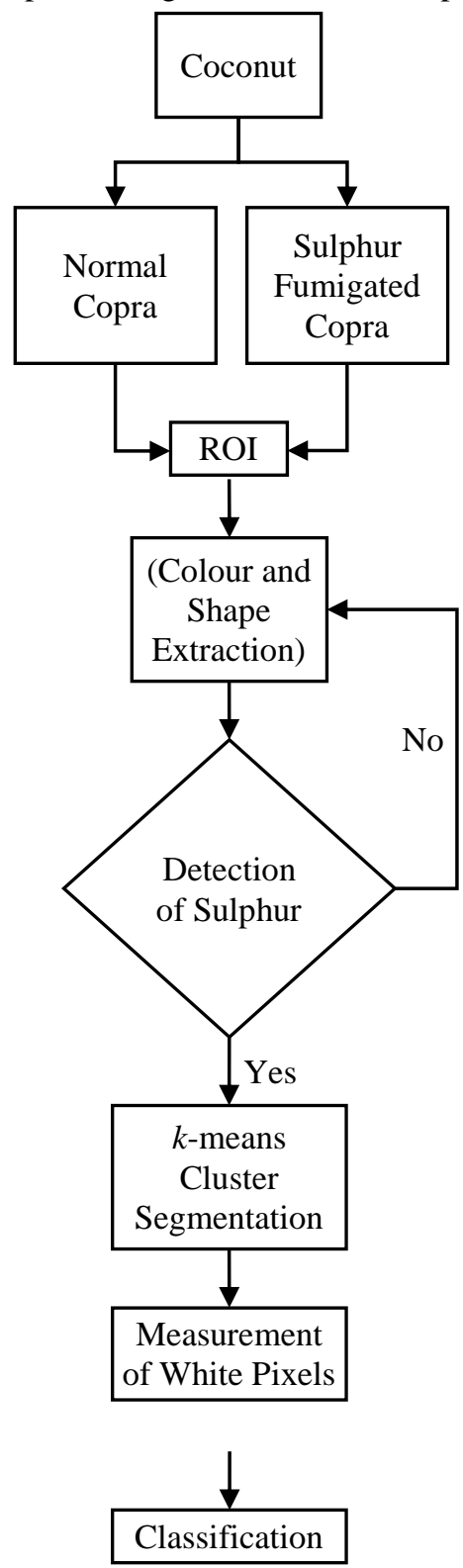

Fig.1. Proposed methodology for identifying sulphur fumigated copra

\section{EXPERIMENTAL RESULTS}

\subsection{ACQUIRED IMAGE}

The images collected from coconut oil industry by using 15 mega pixel camera comprising high quality resolution, are used to analyse the Sulphur fumigated copra clearly and efficiently which is shown in Fig.2. The image is taken under two conditions:

- Image with sulphur fumigated copra and

- Image without sulphur fumigated copra

Image is taken with uniform background to maintain the stability of results.

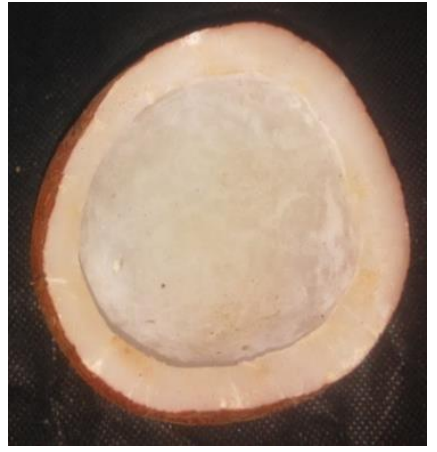

Fig.2. Uniform depiction of Sulphur fumigated copra

\subsection{BACKGROUND REMOVAL BASED ON EXTRACTION OF ROI}

The ROI is white layer in copra. The unwanted background is removed from image focusing on white layer in copra. The background is removed by superimposing white layer of grey image in binary image. The intensity ' 1 ' in binary image represents the copra and intensity ' 0 ' in binary image represents the background. The original image is superimposed on intensity ' 1 ' in binary image.

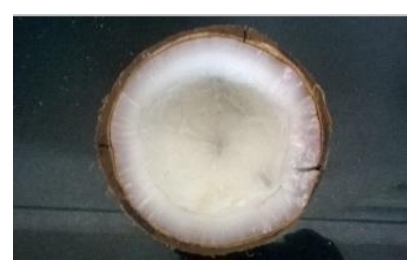

(a)

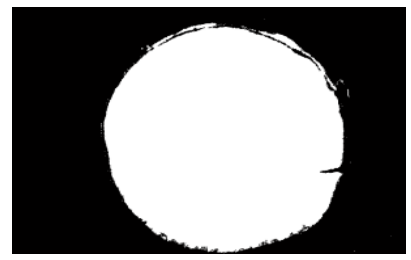

(b)

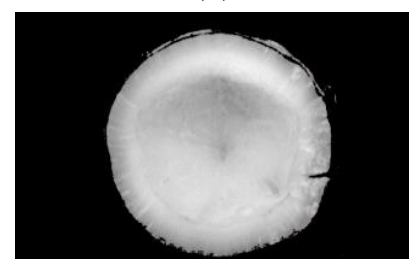

(c)

Fig.3. ROI (a) Original Image (b) Binary Image (c) Superimposed Image

\subsection{SULPHUR CONTENT DETECTION}

Sulphur content can detected either by using RGB color extraction and Major and Minor axis measurement

\subsubsection{RGB Colour Extraction in Copra:}

The sulphur copra has appearance of white and normal dried copra has brownish shade. RGB colour extraction shows the difference in sulphur added copra and normal copra. The combination of RGB results in white colour. The sulphur added copra includes all three colours with high intensity range. 
Meanwhile the normal copra has more red intensity. The RGB colour feature extracted for sulphur added copra is shown in Table.1.

Table.1. RGB Colour Extraction of Sulphur Fumigated copra

\begin{tabular}{|l|c|c|c|}
\hline \multirow{2}{*}{ Images } & \multicolumn{3}{|c|}{$\begin{array}{c}\text { Maximum Intensity } \\
\text { Value of RGB }\end{array}$} \\
\cline { 2 - 4 } & Red & Green & Blue \\
\hline IMG1 & 254 & 254 & 255 \\
\hline IMG2 & 235 & 236 & 241 \\
\hline IMG3 & 243 & 236 & 235 \\
\hline IMG4 & 245 & 241 & 216 \\
\hline IMG5 & 224 & 214 & 205 \\
\hline
\end{tabular}

The Table.1 explains RGB Colour Extraction of Sulphur added Copra which has high intensity range for all three colours (RGB). All the colour ranges are high which exist between 210 and 254. So RGB colour extraction is done for normal copra.

Table.2. RGB Colour Extraction of Normal Copra

\begin{tabular}{|l|c|c|c|}
\hline \multirow{2}{*}{ Images } & \multicolumn{3}{|c|}{$\begin{array}{c}\text { Maximum Intensity } \\
\text { Value of RGB }\end{array}$} \\
\cline { 2 - 4 } & Red & Green & Blue \\
\hline IMG1 & 188 & 158 & 131 \\
\hline IMG2 & 206 & 181 & 177 \\
\hline IMG3 & 205 & 194 & 181 \\
\hline IMG4 & 217 & 207 & 205 \\
\hline IMG5 & 197 & 195 & 186 \\
\hline
\end{tabular}

The Table. 2 dealt about that normal copra has high red colour intensity compared to green and blue intensity. The red intensity is compared with blue and green which is extremely high. The RGB colour extraction differentiates the sulphur fumigated and normal copra by calculating the mean of RGB in test images.

\subsubsection{Major and Minor Axis Measurement:}

Initially the copra is subjected to drying. Drying is carried out by exposing copra in tray drier (Food Processing Lab) to maintain the temperature constantly, since field temperature changes every day. The drying process is done to analyse the physical and chemical changes in the product.

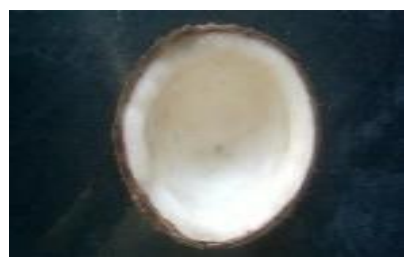

(a)

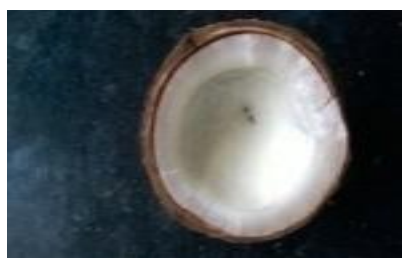

(c)

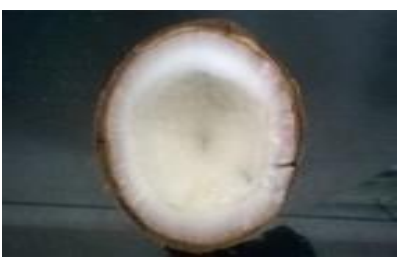

(b)

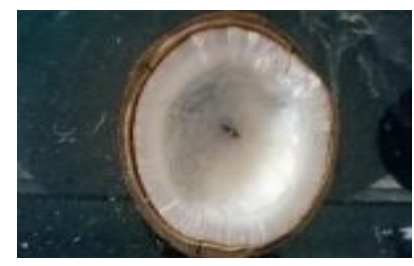

(d)

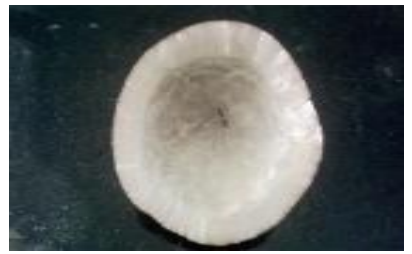

(e)

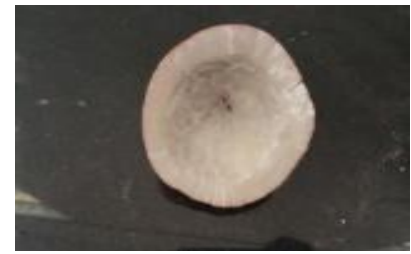

(f)
Fig.4. Changes of copra samples

The Fig.4 defines the changes of a copra sample as a function of drying time (a) Initial stage of coconut (b) dried coconut after 2 hours (c) after 4 hours (d) after 6 hours (e) shell removal copra after 2 hours (f) shell removal copra after 4 hours.

The quality values are changed by the amount of water content. The water content is reduced over dryness of product. The decreasing moisture content of copra decreased an effective influence on its density. The copra dried under $60^{\circ} \mathrm{C}$ at regular interval of 2 hours is shown in Fig.3. The shape of copra is reduced at every stage of drying. The decreasing rate of shape is measured for sulphur added copra and normal copra. The decreasing rate of sulphur added copra is fast compared to normal copra.

The major and minor axes are diameters (lines through the Centre) of the object in image. Each axis cuts the other into two equal parts, and each axis crosses the other at right angles. The centroid of image is found, such that the longest and shortest diameter passes through the centroid. The centroid of image is shown in Fig.4.

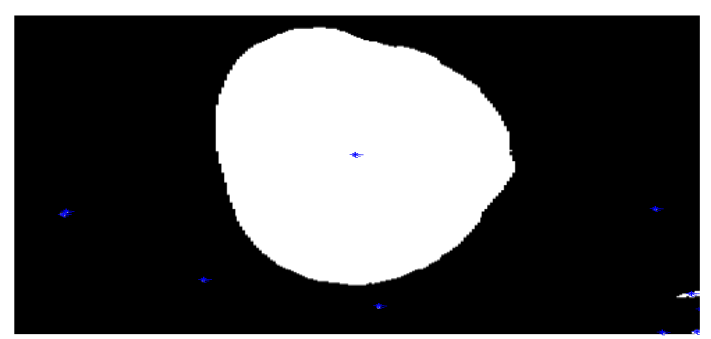

Fig.5. Centroid Marked Copra

The Fig.4 deals with measurement of major axis and minor axis. To find out both axes, the centroids should be found, that both major and minor axis passed through the centroid.

Table.3. Major Axis of sulphureted Copra

\begin{tabular}{|c|c|c|c|c|c|}
\hline \multirow{2}{*}{ IMG } & \multicolumn{5}{|c|}{ Drying Hours } \\
\cline { 2 - 6 } & 2 hours & 4 hours & 6 hours & 10 hours & Dec (\%) \\
\hline I1 & 220.3 & 157.3 & 139.3 & 101.5 & 36.6 \\
\hline I2 & 201.9 & 196 & 157 & 102 & 21.7 \\
\hline I3 & 215 & 213.4 & 165.2 & 136.2 & 23.26 \\
\hline I4 & 216 & 215.5 & 195 & 168 & 17.45 \\
\hline
\end{tabular}

The Table. 3 explains about decreasing percentage of major axis when the sulphur is fumigated. Percentage of drying the sulphur fumigated copra is higher than $15 \%$ (i.e. higher than major axis of normal copra) 
Table.4. Major Axis of Normal Copra

\begin{tabular}{|c|c|c|c|c|c|}
\hline \multirow{2}{*}{ Img } & \multicolumn{5}{|c|}{ Drying Hours } \\
\cline { 2 - 7 } & $\mathbf{2}$ hours & 4 hours & 6 hours & $\mathbf{1 0}$ hours & Dec (\%) \\
\hline I1 & 245 & 233 & 219 & 172.5 & 10.61 \\
\hline I2 & 195 & 185 & 171 & 153 & 12.3 \\
\hline I3 & 178.45 & 173.4 & 161.5 & 150.3 & 9.4 \\
\hline I4 & 226 & 213 & 209 & 179 & 10.61 \\
\hline
\end{tabular}

The Table. 4 explains about the decreasing percentage of major axis of normal copra which is less than $15 \%$ and make a huge difference comparing to major axis of sulphur fumigated copra.

Table.5. Minor axis of sulphureted copra

\begin{tabular}{|c|c|c|c|c|c|}
\hline \multirow{2}{*}{ IMG } & \multicolumn{5}{|c|}{ Drying Hours } \\
\cline { 2 - 6 } & 2 hours & 4 hours & 6 hours & 10 hours & Dec (\%) \\
\hline I1 & 122.6 & 120.6 & 100 & 98.5 & 18.35 \\
\hline I2 & 106.3 & 101.2 & 91.2 & 88.9 & 14.20 \\
\hline I3 & 127.0 & 112.0 & 103.0 & 102.0 & 19.68 \\
\hline I4 & 120.8 & 105.4 & 104 & 95.6 & 13.68 \\
\hline
\end{tabular}

Table.6. Minor axis of Normal copra

\begin{tabular}{|c|c|c|c|c|c|}
\hline \multirow{2}{*}{ IMG } & \multicolumn{5}{|c|}{ Drying Hours } \\
\cline { 2 - 6 } & 2 hours & 4 hours & 6 hours & 10 hours & Dec (\%) \\
\hline I1 & 121.2 & 110.2 & 106 & 102 & 12.31 \\
\hline I2 & 112 & 107 & 102 & 98 & 1.59 \\
\hline I3 & 122.2 & 112.4 & 108.4 & 103.2 & 9.9 \\
\hline I4 & 120 & 119 & 116 & 108 & 3.335 \\
\hline
\end{tabular}

The Table. 5 and Table. 6 describe the minor axis of normal and sulphur fumigated copra. The decreasing percentage is high in sulphur fumigated copra compared to the normal copra.

If decreasing rate of major axis is above $15 \%$ and decreasing rate of minor axis is above $14 \%$, presence of sulphur can be identified. The normal copra has decreasing percentage below $14 \%$ in major axis and below $13 \%$ in minor axis. The detection of sulphur is made by major and minor axis passes through the centroid of copra image.

Table.7. Percentage Calculation

\begin{tabular}{|c|c|c|}
\hline $\begin{array}{c}\text { Decreasing rate of } \\
\text { major axis }(\%)\end{array}$ & $\begin{array}{c}\text { Decreasing rate of } \\
\text { minor axis (\%) }\end{array}$ & Copra \\
\hline $15 \%$ and above & $14 \%$ and above & S-Copra \\
\hline $5 \%-14 \%$ & $1 \%-13 \%$ & N-Copra \\
\hline
\end{tabular}

\subsection{SEGMENTATION OF COPRA USING K-MEANS CLUSTERING ALGORITHM}

The amount of sulphur present in copra is to be specified. To measure the percentage of sulphur, $k$-means clustering segmentation is used to segment the sulphur patch. The region of segmented sulphur patch is measured to determine the percentage of sulphur fumigation in copra. The $k$-means clustering algorithm is used for clustering the image into different clusters with each cluster of different properties. In the proposed work, sulphur added copra is clustered into two clusters of sulphur patch region and sulphur free region. In the proposed work the $k$-means clustering segments the copra into sulphur patched region and sulphur free region. The segmented sulphur regions is shown in Fig.5.

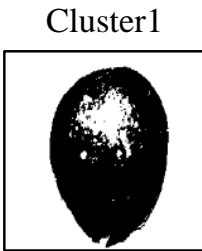

Cluster1

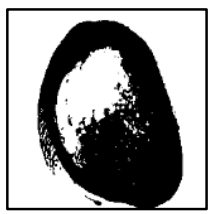

Fig.6. Result of $k$-means Clustering $(k=2)$

\subsection{AREA MEASUREMENT OF SULPHUR PATCH}

The classification of the sulphur copra into different levels is based on feature extraction. The number of white pixel is calculated in image to classify the sulphur copra at different level. The area of white pixel is calculated from the segmented sulphur patch of copra. The sulphur patch is segmented by $k$-means clustering algorithm and it results in binary image. The percentage of sulphur patch present in copra is determined by calculating white pixel from segmented patch and white pixel from original image. The total area of copra is measured to determine the percentage of sulphur present in copra by comparing segmented sulphur patch area.

Table.8. Area Measurement

\begin{tabular}{|c|c|c|}
\hline $\begin{array}{c}\text { Area of sulphur } \\
\text { fumigated }\end{array}$ & $\begin{array}{c}\text { Total area } \\
\text { of copra }\end{array}$ & $\begin{array}{c}\text { Percentage of } \\
\text { sulphur }\end{array}$ \\
\hline 193545 & 195769 & $94 \%$ \\
\hline 547000 & 605080 & $90 \%$ \\
\hline 863812 & 946252 & $87 \%$ \\
\hline 178965 & 374802 & $47 \%$ \\
\hline 283158 & 325860 & $84 \%$ \\
\hline 166020 & 233296 & $71 \%$ \\
\hline
\end{tabular}

By calculating the percentage of sulphur present in copra the sulphur fumigated level is determined as shown in Table.8. The sulphur fumigation level is divided into three levels such as low sulphur region, medium sulphur region and high sulphur region. Low sulphur region in which the area of sulphur patch segmented from copra is less and it is below $40 \%$. The medium sulphur region ranges from $(41 \%-70 \%)$. The excessive sulphur region ranges from $(71 \%-100 \%)$.

\subsection{K-NN CLASSIFIER}

An object is classified by majority vote of its neighbours, with the object being assigned to the class most common among its $\mathrm{k}$ 
nearest neighbours ( $k$ is a positive integer, typically small). The area is extracted and samples trained are 45 and tested samples are 15 . The $k$-NN classification has the accuracy of $86 \%$ with 13 correctly classified samples and 2 wrongly classified samples. The classification of sulphur copra into three regions is performed and it has the accuracy of $86 \%$.

Table.9. Accuracy Measurement

\begin{tabular}{|c|c|}
\hline Parameters & Values \\
\hline Feature extracted & 1 \\
\hline Samples trained & 45 \\
\hline Samples tested & 15 \\
\hline True positive & 13 \\
\hline False positive & 2 \\
\hline True negative & 7 \\
\hline False negative & 1 \\
\hline Accuracy & $86 \%$ \\
\hline Sensitivity & $93 \%$ \\
\hline Specificity & $77 \%$ \\
\hline
\end{tabular}

\section{CONCLUSIONS}

The fumigation of sulphur in copra is one of the leading causes of respiratory diseases and even cause for death. Hence the early detection and identification is necessary. Thus the proposed method to detect the presence and percentage of sulphur in copra is quantified. The RGB colour model extraction and shape feature extraction of drying copra at regular intervals is performed to detect the sulphur present in copra. The segmentation using $k$ means clustering is proposed for copra images to segment the sulphur patch from copra. The $k$-means clustering gives effective segmentation result to segment the sulphur patch from copra. It reduces the computational complexity by using high speed clustering using $k$-means algorithm. The percentages of sulphur present in copra are performed by extracting the white pixel from the segmented image using area measurement. The classifier used to classify the copra in three different regions (low sulphur added region, medium sulphur added region, high sulphur added region) is based on the percentage of sulphur fumigated area. Initially the $k$-NN was trained with the percentage of area measured. Then the test image is given to the $k-\mathrm{NN}$ to test the classification accuracy. The overall classification accuracy obtained is $86 \%$. In future various techniques can be used for extracting features in order to improve classification accuracy.

\section{REFERENCES}

[1] Adel Bakhshipour, "Vision based Features in Moisture Content Measurement during Raisin Production", World Applied Sciences Journal, Vol. 7, No. 7, pp. 860-869, 2012.

[2] Alok Mishra, "The Quality Identification of Fruits in Image Processing using MATLAB", International Journal of Research in Engineering and Technology, Vol. 3, No. 10, pp. 123-129, 2014.
[3] S. Arivazhagan, "Fruit Recognition using Colour and Texture Features", Journal of Emerging Trends in Computing and Information Sciences, Vol. 2, No. 2, pp. 9094, 2010.

[4] P. Deepa and S.N. Geethalakshmi, "Comparative Analysis of Feature Extraction Methods for Fruit Grading Classifications", International Association of Scientific Innovation and Research, Vol. 2, No. 9, pp. 221-225, 2013.

[5] Devrim Unay and Bernard Gosselin, "Automatic Grading of Bi-Coloured Apples by Multispectral Machine Vision", Computers and Electronics in Agriculture, Vol. 75, No. 1, pp. 204-212, 2010.

[6] V. Elamaran, "FPGA Implementation of Point Processes using Xilinx System Generator", Journal of Theoretical and Applied Information Technology, Vol. 41, No. 2, pp. 201 206, 2012.

[7] Kiran Wagh, "Quality Inspection and Grading of Mangoes by Computer Vision and Image Analysis", Journal of Engineering Research and Applications, Vol. 3, No. 5, pp. 1208-1213, 2015.

[8] V. Leemansa, "On-line Fruit Grading According to their External Quality using Machine Vision", Biosystems Engineering, Vol. 83, No. 4, pp. 397-404, 2002.

[9] Monika Jhuria, "Image Processing for Smart Farming: Detection of Diseases and Fruit Grading", Proceedings of $2^{\text {nd }}$ IEEE International Conference on Image Information Processing, pp. 521-526, 2013.

[10] Neha. P. Raut, "FPGA Implementation for Image Processing Algorithms Using Xilinx System Generator", IOSR Journal of VLSI and Signal Processing, Vol. 2, No. 4, pp. 26-36, 2013.

[11] U. Pavan Kumar and P. Padmaja, "Image Enhancement Using Adaptive Filtering", International Journal of Engineering Trends and Technology, Vol. 6, No. 1, pp. 1-4, 2013.

[12] Shiv Ram Dubey and A.S. Jalal, "Detection and Classification of Apple Fruit Diseases using Complete Local Binary Patterns", Proceedings of IEEE International Conference on Computer and Communication Technology, pp. 44-58, 2012.

[13] Sima Kumari and Neelamegam, "Analysis of Apple Fruit Diseases using Neural Network", Research Journal of Pharmaceutical, Biological and Chemical Science, Vol. 2, No. 2, pp. 641-646, 2015.

[14] D.F. Specht, "Experience with Adaptive Probabilistic Neural Network and Adaptive General Regression Neural Network", Proceedings of IEEE International Conference on Neural Networks, pp. 1203-208, 1994.

[15] Tomas U. Ganiron, "Size Properties of Mangoes using Image Analysis", International Journal of Bio-Science and Bio-Technology, Vol. 6, No. 2, pp. 31-42, 2014.

[16] Vani Ashok "Automatic Quality Evaluation of Fruit Using Probabilistic Neural Network Approach”, Proceedings of International Conference on Contemporary Computing and Informatics, pp. 308-311, 2014.

[17] D. Vilas Sadegaonkar and Kiran H. Wagh, "Improving Quality of Apple using Computer Vision and Image Processing based Grading System", International Journal of Science and Research, Vol. 4, No. 4, pp. 543-546, 2013. 